\title{
On the cosmic ray spectrum from type II Supernovae expanding in their red giant presupernova wind
}

\author{
Martina Cardillo \\ INAF/Osservatorio Astrofisico di Arcetri, Largo E. Fermi, 5, 50125 Firenze, Italy
}

\begin{abstract}
While from the energetic point of view SNRs are viable sources of Galactic CRs, the issue of whether they can accelerate protons up to $\mathrm{PeV}$ remains unsolved. Here we discuss particle acceleration at the forward shock of SN and discuss the possibility that the escaping particle current may excite a non-resonant instability that in turn leads to the formation of resonant modes confining particles close to the shock and increasing the maximum energy. This mechanism works throughout the expansion of the SN explosion, from the ejecta dominated (ED) to the Sedov-Taylor (ST) phase. Because of their higher explosion rate,we focus on type II SNae expanding in the slow, dense red supergiant wind. When the explosion occurs in such winds, the transition between the ED and the ST phase is likely to take place within a few tens of years. As a result, the spectrum of accelerated particles shows a break in the slope, at the maximum energy $\left(\mathrm{E}_{M}\right)$ achieved at the beginning of the ST phase. Above this energy, the spectrum becomes steeper but remains a power law than developing an exponential cutoff. We show that for type II SNae typical parameters, proton $\mathrm{E}_{M}$ can easily reach PeV energies, confirming that type II SNRs are the best candidate sources for CRs at the knee. We have tried to fit KASCADE-Grande, ARGO -YBJ and YAC1-Tibet Array data with our model but we could not find any parameter combination that could explain all data sets. Indeed the recent measurement of the proton and helium spectra in the knee region, with the ARGO-YBJ and YAC1-Tibet Array, has made the situation very confused. These measurements suggest that the knee in the light component is at $650 \mathrm{TeV}$, appreciably below the overall spectrum knee. This finding would resolve the problem of reaching very high energies in SNae, but, on the other hand, it would open a critical issue in the transition region between Galactic and extragalactic CRs.
\end{abstract}

\section{Introduction}

Despite supernova remnants (SNRs) are largely accepted as CRs sources because of their energetics (for reviews see, e.g., [7,12]), the issue of whether particles can be accelerated diffusively at the SN shock to sufficiently high energies is still open for debate. One thing that is clear is that effective magnetic field amplification is necessary in order to obtain energies of the order of the knee in the CR overall spectrum, located at $E_{M} \sim 3 \times 10^{15} \mathrm{eV}$. The resonant instability induced by $\mathrm{CR}$ streaming could allow $E_{M} \sim 10^{3}-10^{4} \mathrm{GeV}[23,24]$, still well below theknee just because of its resonant nature [22,32-34,38]. The pioneering papers of Refs. [25] and [10] showed how nonresonant modes can grow very fast ahead of a SNR shock, starting with a wavenumber $k$ much larger than the particle gyroradius but then forming fluctuations on larger scales allowing resonant scattering. This kind of instability may allow acceleration of particles up to the knee if applied to the case of supernovae expanding in the wind of their red super-giant (RSG) progenitor star [11,30], where the highest energies are reached within a few decades after the SN explosion, namely before the beginning of the ST phase of the explosion.

\footnotetext{
a e-mail: martina@arcetri.astro.it
}

The origin of the knee in the all-particle spectrum of CRs is inextricably connected with the issue of the end of the Galactic CR spectrum and the transition to extragalactic CRs. The extra component considered necessary in Ref. [6] to fit the Auger data [2-4,36] appears to be in good agreement with the proton spectrum as measured by KASCADE-Grande [8] (see also the results of ICETOP [1]). However, both the proton and iron spectra measured by KASCADE-Grande in the energy region $10^{16}-10^{18} \mathrm{eV}$ suggest that either the injection spectra are not cut off exponentially at the maximum energy, or there is some, as yet unknown, class of sources with maximum energy much in excess of the knee energy. Moreover, recently, ARGO-YBJ [18] and YAC1-Tibet Array [21] experiments suggest a knee in the light component at $\sim 650 \mathrm{TeV}$, appreciably below $E_{M} \sim 1 \mathrm{PeV}$, making the situation ever more complex.

In the present paper we focused on the issue of reaching the energy of the knee in particle acceleration during the expansion of a SN shock in the dense region occupied by the wind of a RSG. In particular, we investigated in detail the implications of the so called NonResonant Hybrid (NRH) instability described by Refs. [10, $11,30]$ by computing the maximum energy and the overall particle spectrum produced during the whole SN expansion, both in the ED and ST phases, and comparing it with KASCADE-Grande and ARGO data [15]. 


\section{Model}

\section{Maximum Energy}

In order to understand the NRH instability, it is useful to think in physical terms about the process that may be responsible for particle return to the shock from the upstream region. Let us consider a first generation of particles of energy equal to the current achievable maximum, $E$, moving from downstream to upstream. By definition, upstream there are no waves able to scatter resonantly such particles, hence they are going to move quasi-balistically and escape the system at the speed of light (or fractions of it, depending on the level of anisotropy of the distribution of escaping particles). The current of particles at distance $R$ from the explosion center as due to particles escaped when the shock location was at $r$ can be written as:

$$
\begin{aligned}
j_{C R}(R, r) & =n_{\mathrm{CR}, \mathrm{r}}\left(R, E_{M}(r)\right) e v_{s h}(r) \\
& =e \frac{\xi_{C R} \rho(r) v_{s h}^{3}}{E_{0} \Psi(E)}\left(\frac{r}{R}\right)^{2}
\end{aligned}
$$

with

$$
\Psi(E)= \begin{cases}\left(E_{M} / E_{0}\right) \ln \left(E_{M} / E_{0}\right) & \beta=0 \\ \frac{1+\beta}{\beta}\left(\frac{E_{M}}{E_{0}}\right)^{1+\beta}\left[1-\left(\frac{E_{0}}{E_{M}}\right)^{\beta}\right] & \beta \neq 0 .\end{cases}
$$

In the above expressions we have used the relation between the number density of accelerated particles with energy $E$, $n_{C R}(E)$, at some location $R$ upstream of the shock, and the energy density in accelerated particles at the shock $\xi_{C R} \rho(r) v_{s}^{2}(r)$, where $\xi_{C R}$ is the CR acceleration efficiency. The function $\Psi(E)$ accounts for normalisation of the particle distribution function which is taken to be $f_{s}(E) \propto$ $E^{-(2+\beta)}$ and extending between a minimum energy $E_{0}$, that does not depend on time and a maximum energy $E_{M}$ which does depend on time, as we will see (details in [15]). The wavenumber where the growth is the fastest can be easily estimated using the condition

$$
k_{M} B_{0} \cong \frac{4 \pi}{c} j_{C R},
$$

which corresponds to balance between current and magnetic tension. Numerical simulations show that the saturation of the instability occurs after $N_{t} \sim 5$ efolds [10], namely

$$
\int \gamma_{M} d t=\int\left(k_{M} v_{A}\right) d t \approx 5
$$

where $v_{A}$ is the Alfvén speed in the unperturbed magnetic field $B_{0}$. Using the expression for $k_{M}$ derived in Eq. (3), we can see that it depends on CR current (Eq. (1)) and, consequently, on the density profile. For a supernova expanding in the uniform ISM, one can assume that the ISM density is constant $\rho(R)=\rho_{I S M}$. For a type II supernova, instead, it is often the case that the explosion takes place in the wind produced by a red giant presupernova progenitor star. The density of the wind can be written as $\rho(R) \cong \frac{\dot{M}}{4 \pi R_{0}^{2} V_{w}}\left(\frac{R_{0}}{R}\right)^{2}=\rho\left(R_{0}\right)\left(\frac{R_{0}}{R}\right)^{2}$, where $\dot{M}$ is the rate of mass loss of the red giant and $V_{w}$ is the wind velocity. In the following we will simply write $\rho(R) \propto R^{-m}$, having in mind the two cases of $m=0$, corresponding to a uniform medium, and $m=$ 2, appropriate for expansion in the progenitor wind. Differentiating Eq. (4) with respect to $R$, we obtain an implicit expression for the maximum energy (detailed calculations in [15]):

$$
\Psi(E(R)) \cong \frac{2 e}{(4-m) 5 c E_{0}} \xi_{C R} v_{s}(R)^{2} \sqrt{4 \pi \rho(R) R^{2}} .
$$

In order to provide an estimate of the maximum achievable energy as a function of time, we proceed with modeling the transition from the ED to the ST phase of the SN expansion. Using relations showed in details in Ref. [15], we finally can find maximum energy for type I and type II events. In the case of a $E^{-2}(\beta=0)$ source spectrum (we consider this spectrum to the goal of a simple estimate ), for type I events we can estimate:

$$
\begin{aligned}
E_{M} & \cong \frac{2 e}{10 c} \xi_{C R} v_{0}^{2} \sqrt{4 \pi \rho R_{0}^{2}} \\
& =130\left(\frac{\xi_{C R}}{0.1}\right)\left(\frac{M_{e j}}{M_{\odot}}\right)^{-\frac{2}{3}}\left(\frac{E_{S N}}{10^{51} \mathrm{erg}}\right)\left(\frac{n_{I S M}}{\mathrm{~cm}^{-3}}\right)^{\frac{1}{6}} T e V
\end{aligned}
$$

while for type II:

$$
\begin{aligned}
& E_{M} \cong \frac{2 e}{5 c} \xi_{C R} v_{0}^{2} \sqrt{4 \pi \rho R_{0}^{2}} \\
& \approx 1\left(\frac{\xi_{C R}}{0.1}\right)\left(\frac{M_{e j}}{M_{\odot}}\right)^{-1}\left(\frac{E_{S N}}{10^{51} \mathrm{erg}}\right) \\
& \quad \times\left(\frac{\dot{M}}{10^{-5} M_{\odot} / y r}\right)^{\frac{1}{2}}\left(\frac{V_{w}}{10 \mathrm{~km} / \mathrm{s}}\right)^{-\frac{1}{2}} P e V .
\end{aligned}
$$

It is apparent that for standard parameters pertaining the two types of explosions, type II SNe can reach about one order of magnitude larger maximum energies than type Ia, and in particular that if particles are accelerated and escape as described here, they easily seem to be able to provide proton acceleration up to the knee. It can be shown that $E_{M}$ as derived from Eqs. (5) and (2) changes by about 1 order of magnitude at the transition between ejecta dominated and Sedov phase depending on the source spectral index: spectral indices steeper than 2 lead to lower values of $E_{M}$ compared to the estimate in Eq. (7), while the opposite is true for flatter spectra [15].

\section{Particle spectrum}

For a supernova shock moving with velocity $v_{s h}(t)$ in the surrounding medium (ISM or wind of the progenitor star) the shock radius is $R_{s h}(t)=\int_{0}^{t} d t^{\prime} v_{s h}\left(t^{\prime}\right)$. At each time $t$ we assume that particles with given energy $E_{M}(t) \equiv E$ can escape the accelerator. The number of particles that escape the shock at each given time is related to the current at the 
upstream radius $R$ through:

$$
N_{e s c}(E) d E=\frac{J_{C R}}{e} 4 \pi R^{2} d t,
$$

where $N_{\text {esc }}(E)$ is the number of particles per unit energy, with energy $E=E_{M}(t)$, that are released in the ISM. Using the expression in Eq. (1) for the current and assuming a general power-law dependence of the shock radius on time: $R \propto t^{\lambda}$, which also implies $v_{s} \propto R^{(\lambda-1) / \lambda}$, one can rewrite (details in [15]):

$$
N_{e s c}(E)=\frac{4 \pi \xi_{C R}}{E_{0} S(\lambda, m)} \rho v_{s}^{2} R^{3} \chi(E),
$$

where $\chi(E)=(d / d E)(1 / \Psi(E))$ is a function of $E$ alone, to be computed from Eq. (2). When computing $\chi(E)$ explicitly from Eq. (2) we find:

$$
\chi(E) \approx \frac{d}{d E}\left(\frac{1}{\Psi}\right)=\frac{1}{E_{0}} \begin{cases}\frac{\beta}{1+\beta}\left(\frac{E}{E_{0}}\right)^{-2} & \beta<0 \\ \left(\frac{E}{E_{0}}\right)^{-2}\left[\frac{\left(1+\ln \left(E / E_{0}\right)\right)}{\left(\ln \left(E / E_{0}\right)\right)^{2}}\right] & \beta=0 \\ \beta\left(\frac{E}{E_{0}}\right)^{-(2+\beta)} & \beta>0\end{cases}
$$

where the sign $\approx$ refers to the fact that we have used the assumption $E>>E_{0}$. It is apparent that the power-law dependence of $\chi(E)$ can only be -2 or steeper. Therefore the spectrum of CRs released during the Sedov-Taylor phase is the same as the spectrum of accelerated particles in the source only if the latter is $E^{-2}$ or steeper, while for flatter source spectra $N_{e s c}(E) \propto E^{-2}$.

While in the Sedov-Taylor phase, only the environmental details are important while the ejecta density profile is irrelevant to the spectral slope, during the free expansion phase both density profiles are important in order to determine the position of the breaks and the maximum energy to which the released spectrum extends. This kind of information enters the calculation of the dependence of the shock radius on time, which we expressed as $R \propto t^{\lambda}$.

In the following, we take the value of $\lambda$ appropriate to describe the different evolutionary stages of the SNR from Refs. [16,17,27,35]. This work gives the remnant expansion law during the different stages for generic density profiles of both the SN ejecta $\rho_{e j} \propto R^{-k}$ and the ambient medium $\rho \propto R^{-m}$. Once $\lambda$ is given, from Eqs. (9) and (10) we can derive the spectrum released by the SN during the ED and ST phases as [15]:

$$
N_{e s c}(E) \propto \begin{cases}E^{-(5+4 \epsilon)} & \text { ED phase; } \\ E^{-(2+\epsilon)} & \text { ST phase; }\end{cases}
$$

for Type I SNR expanding in the ISM, and

$$
N_{e s c}(E) \propto \begin{cases}E^{-(4+3 \epsilon)} & \text { ED phase; } \\ & m=2, k=9 \\ E^{-(2+\epsilon)} & \text { ST phase } ;\end{cases}
$$

for Type II SNR expanding in their red supergiant wind. The variable $\varepsilon$ depends on the injection spectrum: $\varepsilon=0$ for an injection index $p_{i n j} \leq 2, \varepsilon=\beta$ for an injection index $p_{i n j}=2+\beta$. This result is very interesting because it shows that the spectrum of escaping CRs integrated over time during the SN shock expansion is a broken power law, with an index close to 2 at low energies (below $E_{M}$ ) and steeper at higher energies. The transition between the two power laws at the maximum energy $E_{M}$ corresponds to the transition between the end of the ED phase, where most mass has already been processed, and the beginning of the ST (adiabatic) phase.

At this point we have to consider the transport of nuclei in the Galaxy. Assuming that the transport has reached a stationary regime and that it is dominated by diffusion and that both injection and spallation occur in a thin disc of size $2 h$, the solution of the transport equation in the Galactic disc can be easily written as follows:

$$
f_{0, \alpha}(E)=\frac{N_{\alpha}(E) \Re}{\pi R_{D}^{2} \mu v} \frac{X(E)}{1+\frac{X(E)}{X_{\alpha}}},
$$

where we introduced $\mathrm{SN}$ explosion rate $\Re$ and the grammage

$$
X(E)=n_{d} \frac{h}{H} v m_{p} \frac{H^{2}}{D(E)} .
$$

Here the injection spectra $N_{\alpha}(E)$ are calculated as discussed but using the parametric form for $R_{s h}, n_{d}$ is the gas density of the Galactic disc, assumed of thickness $2 h$ and radius $R_{D}$. Written in this form, the solution is very clear. At particle momenta for which the grammage is small compared with $X_{\alpha}$ the standard solution is recovered:

$$
f_{0, \alpha}(E)=\frac{N_{\alpha}(E) \Re}{2 \pi R_{D}^{2} H} \frac{H^{2}}{D(E)},
$$

while in the range of momenta at which the solution is spallation-dominated, the observed spectrum reproduces the shape of the injection spectrum. In order to calculate the spectra of CRs at the Earth, an evaluation of the grammage is needed and we use the formulation of Ref. [28] as starting point. Given that the CR spectrum observed at Earth has an energy dependence $E^{-2.65}$ as taken from TRACER and CREAM data [9,37], in our calculations we used a slope of the diffusion coefficient $\delta=2.65-p_{i n j}$, where for $p_{i n j}$ we considered the values 2 and 2.31. In the first case, the diffusion coefficient has a slightly stronger energy dependence than the one used by [28] $\left(E^{0.6}\right)$; in the other case, instead, we use his exact form. For the spallation cross section we adopt the simple formulation of Ref. [20] (details in [15]).

\section{Results}

Having shown that type II SNe are the most likely sources to accelerate protons up to the knee, we focus on this type of explosions and attempt at fitting the all particle spectrum. To this aim we vary the different parameters, including the ejecta density profile. We have seen that the value of $E_{M}$ is determined by the combination of the CR acceleration efficiency, $\xi_{C R}$, and the energetics of the SN, $E_{S N}$. On the other hand, the flux of CRs at the Earth derives from a combination of $\xi_{C R}, E_{S N}$ and the rate of 
SNe, $\Re$. The need to fit the proton spectrum in the knee region imposes an additional constraint on the combination $\xi_{C R} E_{S N} \Re$ (see Fig. 2 in [15]).

We calculated the required efficiency and rates necessary to reach the knee and fit the overall CR flux: for steep spectra at the shock, the escape current is lower and this forces one to have larger SN energetics and larger $\mathrm{CR}$ acceleration efficiencies, which is counterintuitive for a steep spectrum of accelerated particles. For flat $E^{-2}$ spectra at the shock, the spectrum of escaping particles is also flat and $\mathrm{PeV}$ energies can be reached for ordinary parameters of the $\mathrm{SN}$ explosion. Reaching PeV energies is even easier for hard spectra at the shock. Our calculations show that a type II SN with standard energetics $\left(E_{S N}=\right.$ $10^{51} \mathrm{erg}, \xi_{C R}=10 \%$ ) can accelerate CRs up to knee energies, $E_{M} \sim 1 \mathrm{PeV}$. The spectrum obtained with these parameters is shown in Fig. 3 of [15].

It is interesting to appreciate how particle acceleration at $\mathrm{PeV}$ energies typically occurs $\sim 10-30$ years after the $\mathrm{SN}$ explosion, which represents a change of paradigm with respect to the standard SNR paradigm where the highest energies are reached several hundred years after explosion. In this scenario, the probability of catching a PeVatron in action in our own Galaxy (for instance through its gamma ray emission) is very low.

The comparison of the expected spectra of light nuclei with the recent data of KASCADE-Grande and ARGO appears very problematic: while individually both sets of data can be fitted with reasonable values of the SN parameters and $\mathrm{CR}$ acceleration efficiencies, there is no model that can fit both at the same tim (Figs. 5 and 6 of [15]). KASCADE-Grande data require a SN energy $E_{S N}=2 \times 10^{51} \mathrm{erg}$, an efficiency $\xi_{C R}=20 \%$ and a rate of explosion of $\Re=1 / 110 \mathrm{yr}^{-1}$ that lead to a maximum energy $E_{M} \cong 3.7 \mathrm{PeV}$, whereas we can fit ARGO data with $E_{S N}=10^{51} \mathrm{erg}, \Re=1 / 15 y r^{-1}$ and $\xi_{C R} \cong 5.2 \%$ that lead to $E_{M} \cong 507 \mathrm{TeV}$.

The disagreement between these two experiments is a purely experimental problem, that requires a serious and careful assessment of the systematic uncertainties involved in the adopted experimental techniques.

\section{References}

[1] M.G. Aartsen et al., Phys. Rev. D 88, 042004 (2013)

[2] Abbasi, R. et al., Phys. Rev. Lett. 92, 151101 (2004)
[3] Abbasi, R. et al., Phys. Rev. Lett. 100 (2008) 101101

[4] Abraham, J. et al., Phys. Rev. Lett. 104 (2010) 091101

[5] Adriani, O. et al., Science, 332 (2011) 69

[6] Aloisio, R. et al., (2013) arXiv: 1312.7459

[7] Amato, E., JMPD 23 (2014), 1430013

[8] Apel, W., et al., Phys. Rev. 87 (2013) 1101

[9] Ave, M. et al., ApJ 678 (2008) 262

[10] Bell, A.R., MNRAS 353 (2004) 550-558

[11] Bell, A.R. et al., MNRAS 431 (2013) 415-429

[12] Blasi, P., A\&ARv, 21 (2013) 70

[13] Blasi, P., CRPhy 15 (2014) 329

[14] Caprioli D. et al., ApJ 794 (2014) 46

[15] Cardillo, M., Amato, E. \& Blasi, P., Astrop. Phys., 10.1016/j.astropartphys.2015.03.002 (2015)

[16] Chevalier, R.A., ApJ 258 (1982) 790

[17] Chevalier, R.A. et al., ApJ 344 (1989) 332

[18] De Mitri, I. et al., (ARGO-YBJ coll.), Proc. of Frontier Research in Astrophysics, Vulcano (2014)

[19] Di Sciascio, R. et al., Proc. of 33th ICRC, (2013), Rio de Janeiro

[20] Horandel, J.R. et al., Astrop. Phys. 27 (2007) 119

[21] Huang, J. et al., Proc. of 33th ICRC, Rio de Janeiro (2013)

[22] Kulsrud R. \& Pearce W. P., ApJ 156 (1969) 445

[23] Lagage, O. \& Cesarsky, C.J., A\&A 118 (1983) 223

[24] Lagage, O. \& Cesarsky, C.J., ApJ 125 (1983) 249

[25] Lucek, S.G. \& Bell, A.R., MNRAS 314 (2000) 65

[26] Malkov, M.A. \& Drury, O., Rep. Prog. Phys. 64 (2001) 429

[27] Ptuskin, V.S. \& Zirakashvili, V.N., A\&A 429 (2005) 755

[28] Ptuskin, V.S. et al., Astrop. Phys. 31 (2009), 284

[29] Riquelme M.A. et al., ApJ 694, 626 (2009)

[30] Schure, K.M. \& Bell, A.R., MNRAS 435 (2013) 1174

[31] Schure, K.M. \& Bell, A.R., MNRAS 437 (2014) 2802

[32] Skilling J., MNRAS 172 (1975a) 557

[33] Skilling J., MNRAS 173 (1975b) 245

[34] Skilling J., MNRAS 173 (1975c) 255

[35] Truelove, J.K. \& McKee, C.F., ApJ Supp. Series 120 (1999) 299

[36] Tsunesada, Y., ICRC 12 (2011) 67

[37] Yoon, Y.S. et al., ApJ 728 (2011) 122-129

[38] Wentzel D.G., ARA\&A 12 (1974) 71 